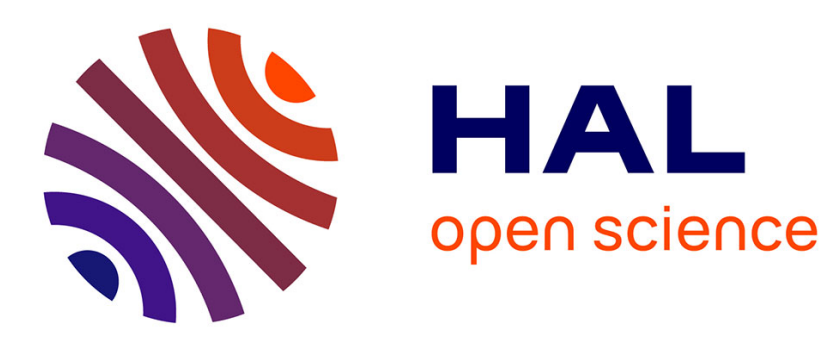

\title{
Anisotropy at the Inner Core Boundary
}

George Helffrich, David Mainprice

\section{- To cite this version:}

George Helffrich, David Mainprice. Anisotropy at the Inner Core Boundary. Geophysical Research Letters, 2019, 46 (21), pp.11959-11967. 10.1029/2019GL083644 . hal-02445502

\section{HAL Id: hal-02445502 \\ https://hal.umontpellier.fr/hal-02445502}

Submitted on 20 Jan 2020

HAL is a multi-disciplinary open access archive for the deposit and dissemination of scientific research documents, whether they are published or not. The documents may come from teaching and research institutions in France or abroad, or from public or private research centers.
L'archive ouverte pluridisciplinaire $\mathbf{H A L}$, est destinée au dépôt et à la diffusion de documents scientifiques de niveau recherche, publiés ou non, émanant des établissements d'enseignement et de recherche français ou étrangers, des laboratoires publics ou privés. 


\section{Geophysical Research Letters}

\author{
RESEARCH LETTER \\ 10.1029/2019GL083644 \\ Key Points: \\ - The isotropy of the surface of the \\ inner core may only be apparent; VTI \\ anisotropy is possible \\ - PKiKP amplitudes in the 75-90 \\ range are explained better by VTI at \\ the ICB with 5-10\% anisotropy than \\ by isotropic models
}

Supporting Information:

- Supporting Information S1

Correspondence to:

G. Helffrich,

george@elsi.jp

Citation:

Helffrich, G., \& Mainprice, D. (2019). Anisotropy at the inner core boundary. Geophysical Research Letters, 46, 11,959-11,967. https://doi.org/10.1029/ 2019GL083644

Received 8 MAY 2019

Accepted 24 AUG 2019

Accepted article online 3 SEP 2019

Published online 15 NOV 2019

(C)2019. American Geophysical Union. All Rights Reserved.

\section{Anisotropy at the Inner Core Boundary}

\author{
G. Helffrich ${ }^{1}\left(\mathbb{D}\right.$ and D. Mainprice ${ }^{2}(\mathbb{D}$ \\ ${ }^{1}$ Earth-Life Science Institute, Tokyo Institute of Technology, Tokyo, Japan, ${ }^{2}$ Géosciences Montpellier UMR CNRS 5243, \\ Université Montpellier 2, Montpellier, France
}

\begin{abstract}
The outermost part of the inner core is isotropic, though the thickness of this layer varies longitudinally, commonly interpreted to be a zone where compaction of the crystallites randomly adhering to the inner core boundary eventually aligns them. We explore the possibility that the surface layer is also anisotropic, but of a form that thwarts detection by travel time observation. If the surface material is hexagonally anisotropic with a vertical symmetry axis (vertically oriented transverse isotropy), $P$ wave travel times through the shallow inner core will not depend on their angle to the Earth's rotation axis. To detect any anisotropy in this layer, we explore PKiKP amplitudes versus distance to determine whether there is a range trend diagnostic of anisotropy. $P$ wave anisotropy levels of $2-10 \%$ can increase reflection amplitudes by a factor of 10 . The trend of PKiKP amplitude with range may be fit with vertically oriented transverse isotropy anisotropy levels of 5-10\%.
\end{abstract}

Plain Language Summary Seismic waves travel through Earth's solid inner core at different speeds in different directions. This happens because the metal crystals in the core line up to give the inner core a grain, like wood. The outermost part of the inner core's surface seems to lack a grain, however, because the speeds in it do not depend on direction. This study explores the possibility that there is a grain to the surface of the inner core too-it is just oriented differently. If the crystals align themselves to all point outward, you will not see changes in speed with direction with the usual seismic waves that travel across the inner core. Waves that reflect from the inner core's surface will seem much brighter, however, if the grains all point outward. It turns out that reflections from the inner core are much brighter than you would expect, lending credence to the idea of texture in it everywhere. As the crystals accumulate on the inner core's surface, they eventually get pressed into the grain orientation that the rest of the inner core has.

\section{Introduction}

The inner core is generally accepted to be anisotropic based on observations made with a variety of seismological methods. Body wave travel times first suggested the inner core's anisotropy (Poupinet et al., 1983), which was subsequently verified by more focused studies (Creager, 1992; Morelli et al., 1986; Shearer, 1994; Song \& Helmberger, 1993). Concurrently, anomalously split normal mode eigenfrequencies also pointed to the structure in the inner core (Giardini et al., 1987; Ritzwoller et al., 1986; Woodhouse et al., 1986). Since then, a plethora of body wave and normal mode studies probed the structure of the inner core's anisotropy; see Tkalčić (2015) for a review.

The inner core's anisotropy is not, however, simple. Among its complexities is the quasi-hemispheric dichotomy in the anisotropy's strength (Tanaka \& Hamaguchi, 1997) and radial strength (Creager, 1999; Song \& Helmberger, 1998), which itself varies hemispherically (Creager, 2000; Garcia \& Souriau, 2000). Essentially, an isotropic layer, whose thickness varies with longitude, overlies the bulk anisotropy of the deeper inner core. Explanations for the longitudinal structure range from varying heat flow at the core-mantle boundary, affecting the crystallizing inner core (Sumita \& Olson, 2002) to lopsided growth due to a translational mode (Alboussière et al., 2010; Monnereau et al., 2010), but are unimportant for the purposes of this study. Rather, we focus on the solidification texturing that takes place after adhesion of a crystallite to the inner core surface (Deguen, 2012) and orients the solids to the inner core's bulk anisotropy.

The intrinsic anisotropy of a mineral is usually quite large compared to the bulk anisotropy of an aggregate (Barruol \& Mainprice, 1993). Hexagonal closest-packed (HCP) iron is widely believed to be the phase crystallizing from the outer core liquid to form the inner core; consequently, its elastic properties are a common target of state-of-the-art first-principles computational studies (Li et al., 2018; Steinle-Neumann et al., 2001; 


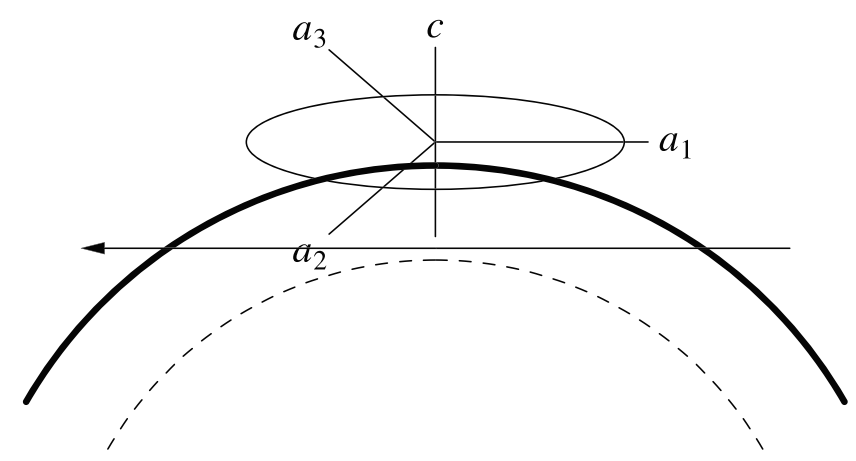

Figure 1. Sketch of the concept of an anisotropic inner core boundary. Inner core boundary surface is the solid line, and the layer with vertically oriented transverse isotropy is bounded below by the dashed line. A $P$ wave such as PKPdf traveling through the anisotropic layer (arrow) will not undergo any directional change of wave speed, appearing to be isotropic. The sketch shows the equatorial plane and $a$ and $c$ axes of the layer's hexagonal symmetry.

Stixrude \& Cohen, 1995). For example, $P$ anisotropy (in the axial to equatorial directional wave speeds in hexagonal symmetry) ranges from 3-22\% among the three cited single-crystal values. This raises the possibility that the surface of the inner core is actually substantially anisotropic but organized with the crystallite symmetry axes radial (vertically oriented transverse isotropy or VTI). In this orientation, the $P$ waves traditionally used to study inner core anisotropy, PKPbc, and PKPdf (also known as PKIKP) would traverse the inner core in the equatorial plane of the hexagonal system and would not be subject to travel time variation dependence on the angle with respect to the Earth's spin axis (Figure 1). Hence, the effect of the anisotropy on travel times would be undetectable. Lythgoe and Deuss (2015) also noted this in their study of center frequency shifts of inner core-sensitive normal modes.

Anisotropy also affects the reflection coefficients off of boundaries in layered media, however. One way to see how this arises is from the consequences of propagation in anisotropic media: the wave vector (ray direction) differs from the energy flux direction. When a boundary is reached, its reflectivity changes abruptly when a transmitted wave is no longer able to penetrate the boundary. In the isotropic case, this is when the wave vector becomes parallel to the interface, but in anisotropic media this is when the energy flux parallels the interface. This leads to a different angle dependence of reflection amplitude than the isotropic case, and to different amplitudes at any particular incidence angle.

In this study we demonstrate how strong this effect is by examining the $P$ wave reflection at the inner core boundary (ICB), PKiKP. We find that order-of-magnitude amplitude enhancement is likely in some incidence angle ranges, which provides an alternative explanation to unexpectedly strong $P$ wave reflections off of the ICB. For reflected wave amplitudes, anisotropy is a first-order effect. We show that weak single-crystal anisotropy is capable of producing this strong of a reflectivity variation. Consequently, the ICB's surface might not be isotropic, but rather markedly anisotropic with an orientation that obscures a travel time signature.

\section{Methods}

The $P$ wave anisotropy is fairly weak and cylindrically symmetric (Creager, 1992), so we use the parameterization of Thomsen (1986) to characterize it (an approximate theory applicable to up to 20\% anisotropy). The five independent elastic constants for hexagonal symmetry are related to three nondimensional parameters $\epsilon, \delta$, and $\gamma$ and two characteristic wave speeds $\left(V_{P 0}\right.$ and $\left.V_{S 0}\right)$ along the symmetry axis. Explicitly, the wave speed dependence on angle with respect to the symmetry axis $\theta$ is

$$
\begin{aligned}
V_{P}(\theta) & =V_{P 0}\left[1+\delta \sin ^{2} \theta \cos ^{2} \theta+\epsilon \sin ^{4} \theta\right] \\
V_{S V}(\theta) & =V_{S 0}\left[1+(\epsilon-\delta)\left(V_{P 0} / V_{S 0}\right)^{2} \sin ^{2} \theta \cos ^{2} \theta\right] . \\
V_{S H}(\theta) & =V_{S 0}\left[1+\gamma \sin ^{2} \theta\right]
\end{aligned}
$$

$\epsilon$ and $\delta$ control the axial to equatorial variation in $P$ wave speed ( $\epsilon$ is the axial-to-equatorial anisotropy) and the SV wave speed, whereas $\gamma$ affects only the SH wave speed. To relate the quantitites to the components 


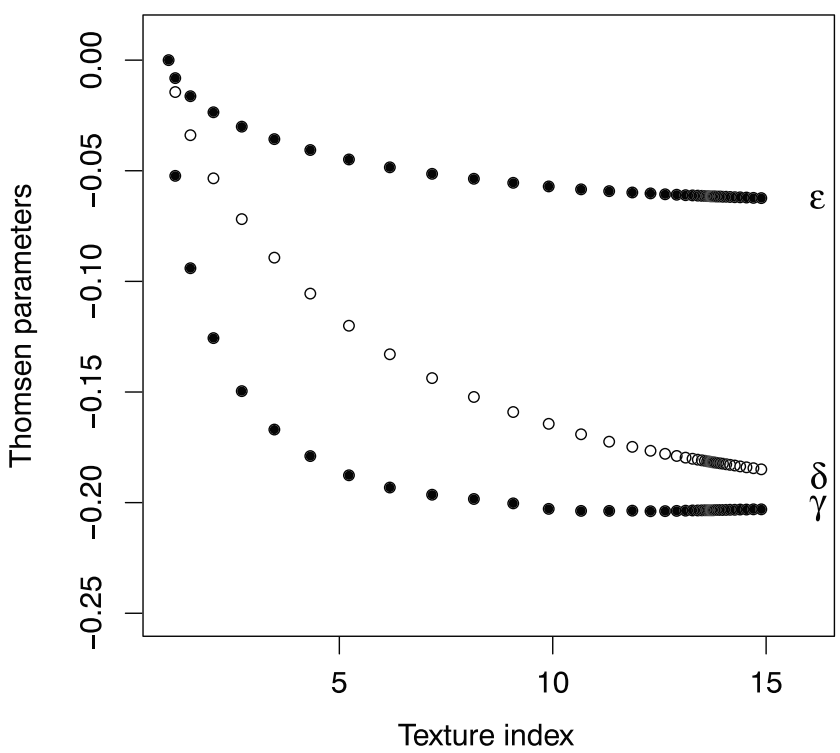

Figure 2. Evolution of Thomsen parameters $\epsilon, \delta$, and $\gamma$ for Mg with increasing uniaxial compression, parameterized by the texture index. The anisotropy parameters appear to approach asymptotic limits. Over the range shown mean $\delta / \epsilon$ and $\gamma / \epsilon$ are 2.9 and 3.4, respectively.

of the Voigt form of the elastic tensor $C_{i j}$,

$$
\begin{aligned}
V_{P 0} & =\sqrt{C_{33} / \rho} \\
V_{S 0} & =\sqrt{C_{44} / \rho} \\
\epsilon & =\frac{C_{11}-C_{33}}{2 C_{33}} \\
\delta & =\frac{\left(C_{13}-C_{44}\right)^{2}-\left(C_{33}-C_{44}\right)^{2}}{2 C_{33}\left(C_{33}-C_{44}\right)} \\
\gamma & =\frac{C_{66}-C_{44}}{2 C_{44}}
\end{aligned}
$$

In hexagonal symmetry (with $c \| x_{3}$ ), the Voigt elastic tensor is

$$
C_{i j}=\left[\begin{array}{cccccc}
C_{11} & \left(C_{11}-2 C_{66}\right) & C_{13} & & \\
\left(C_{11}-2 C_{66}\right) & C_{11} & C_{13} & & \\
C_{13} & C_{13} & C_{33} & & \\
& & & C_{44} & \\
& & & & C_{44} & \\
& & & & & C_{66}
\end{array}\right] .
$$

To derive $C_{i j}$ values from the isotropic wave speeds of the solid inner core, we identify $V_{P}$ with a $P$ wave traveling normal to the hexagonal symmetry axis and $V_{S}$ with a similarly traveling SV wave and use (1) and (2) to solve for $C$. See supporting information Table S1 for the isotropic values used in equations (1) and (2). Anisotropic transmission and reflection coefficients are obtained using methods from Malehmir and Schmitt (2016). In all cases, we assume that the layer boundary is normal to $x_{3}$. We simulate the ICB reflection of PKiKP by placing the hexagonal symmetry axis in the SV ray plane. We validated the reflection coefficient methodology by reproducing the reflection coefficient trend with angle for PKiKP when $\epsilon=\delta=\gamma=0$ and $V_{P 0}$ and $V_{S 0}$ the Preliminary Reference Earth Model (PREM) inner core surface wave speeds (Dziewonski \& Anderson, 1981), with $V_{P 0}$ the base of the outer core's wave speed and $C_{44} / C_{33}=C_{66} / C_{33}=1 \times 10^{-6}$ for the liquid.

We used the robust visco-plastic self-consistent code originally developed for HCP metals (Lebensohn \& Tomé, 1993) using critical resolved shear values (supporting information Table S2) measured for pure magnesium at temperature $T / T_{\text {melt }}=0.7$ (Chapuis \& Driver, 2011). In HCP metals the $c / a$ ratio controls their 


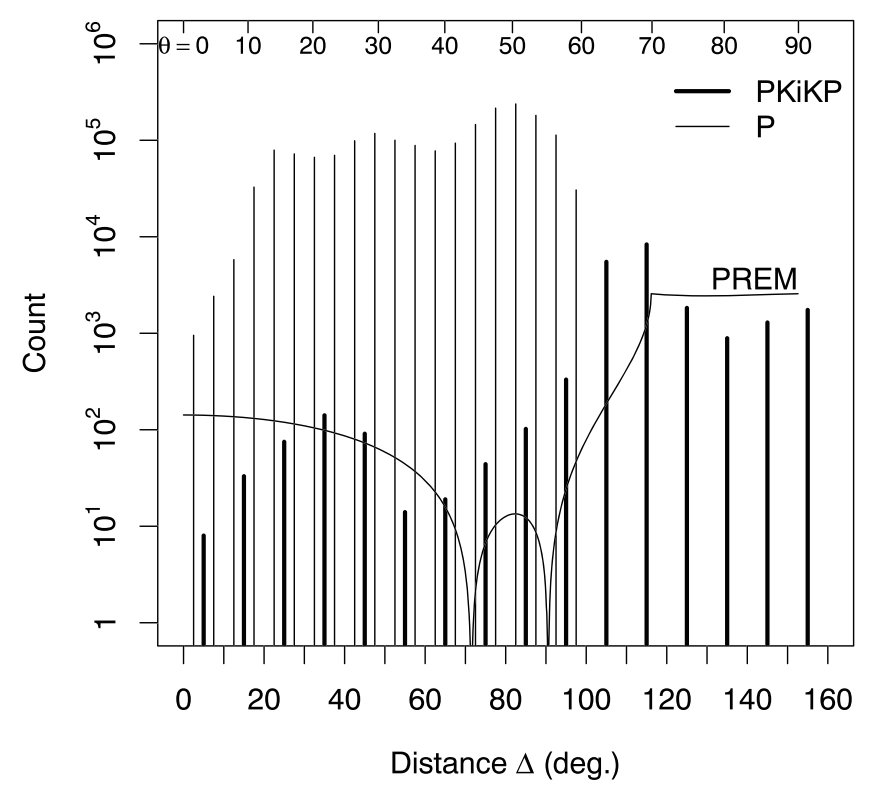

Figure 3. Histograms of reporting frequencies of $P$ and PKiKP arrivals to the International Seismological Centre for events of magnitude 5 and greater, as a proxy for the range dependence of PKiKP amplitudes. The level of $P$ reports is relatively uniform in the range $20-90^{\circ}$, indicating that geometric and geographic biases affect this proxy for PKiKP amplitudes $<20^{\circ}$. Beyond $120^{\circ}$, reporting levels for PKiKP are also relatively constant as the reflection coefficients would indicate; the line shows the theoretical PREM PKiKP amplitude. Between $50^{\circ}$ and $120^{\circ}$ there is significant deviation in PKiKP amplitude from either uniform or PREM-predicted amplitudes. PREM = Preliminary Reference Earth Model.

dislocation slip geometry. Magnesium has the same $c / a$ ratio as HCP iron at inner core conditions (Li et al., 2018). The visco-plastic self-consistent code simulations use axial compression, as the target symmetry is hexagonal to an equivalent strain of one. (We use the adiabatic elastic constants to derive the anisotropy, but note that the anisotropy of the isothermal elastic constants is lower.) Axial compression mimics the process of compaction of crystallites at the surface of the ICB. As expected, the slip activity is dominated by basal

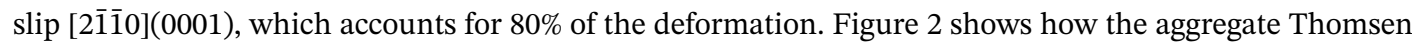
parameters $\epsilon, \delta$, and $\gamma$, calculated from the $C_{i j}$ values using (1) and (2), evolve as a function of texture index (Mainprice et al., 2015), which yields a rule of thumb for anisotropy developed from texturing that if the $P$ wave anisotropy is $\epsilon$, then $\delta \approx 2.9 \times \epsilon$ and $\gamma \approx 3.4 \times \epsilon$.

In order to compare predicted PKiKP amplitudes with data, we use records of PKiKP arrivals cataloged by the International Seismological Centre. Though the International Seismological Centre data includes amplitude information, it is not uniformly reported and is therefore subject to geographic bias arising from recording network operating practices. Hence, we use the frequency of reported observations of PKiKP with distance as a rough gauge of amplitude. We select all earthquakes in a 13-month period starting in October 2010 with magnitude $>5$ listing PKiKP arrivals, which yields about $2 \times 10^{4}$ observations. To account for source-receiver bias in the PKiKP observations, we also select all $\mathrm{P}$ arrivals for the same interval and magnitude cutoff, about $2 \times 10^{6}$ observations. $P$ and PKiKP are jointly present in the range $\Delta$ of $0-100^{\circ}$, which helps to assess the $\sin \Delta$ area bias due to the spherical Earth as well. Figure 3 depicts histograms of $P$ and PKiKP reports; further, maps and diagnostic plots of geographic bias may be found in the supplementary information. The bottoming point maps and histograms show the expected biases to the Northern Hemisphere and the Eastern Hemisphere and to subduction zone sources. Intermediate-range (50-100 $)$ PKiKP reflections mostly occur in the Eastern Hemisphere.

The histograms in Figure 3 reveal that $P$ reports are relatively uniform in the range of $20-90^{\circ}$, suggesting no geometric bias in that range. Below $20^{\circ}$, geometric biases probably act to reduce reporting frequency, and above $90^{\circ} \mathrm{P}$ observations probably also decrease due to the near-simultaneous arrival of $P$ and $\mathrm{PcP}$ (the $P$ core reflection), hindering identification. The PKiKP arrivals below $25^{\circ}$ presumably reflect the same geographic and geometric biases as $P$ and will not be used in the following analyses. Beyond $\sim 120^{\circ}$, PKiKP reporting frequencies are relatively constant, as expected from the calculated reflection coefficient for PREM. See the 

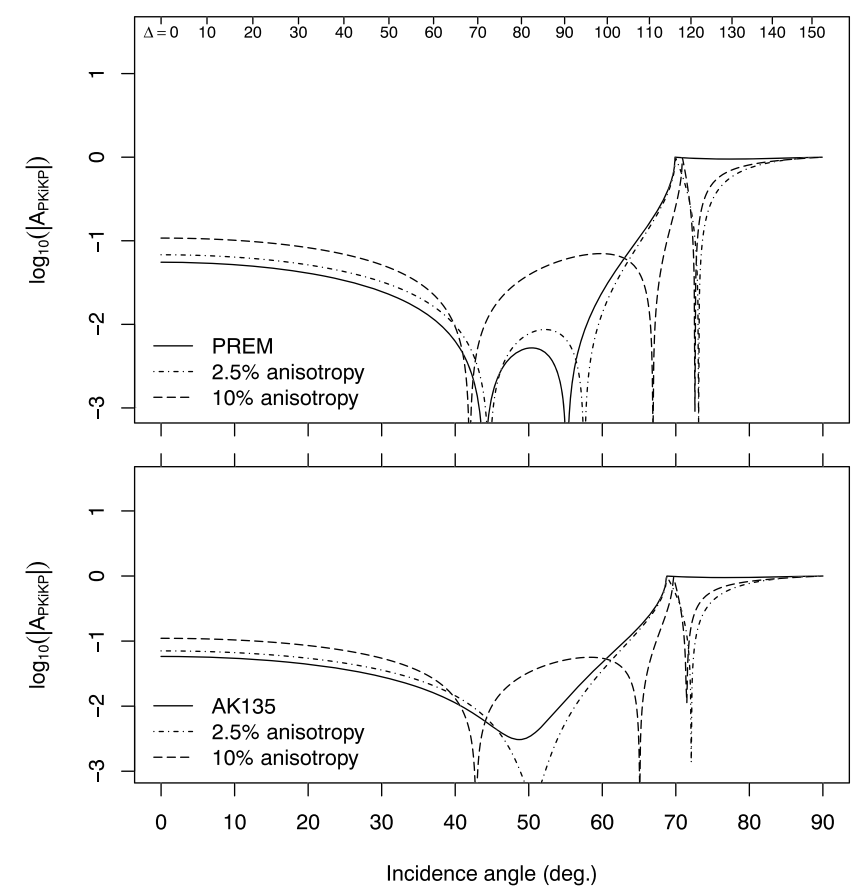

Figure 4. PKiKP reflection coefficient amplitude for isotropy and different levels of axial-to-equatorial $P$ wave anisotropy. Effect on PREM PKiKP amplitude shown at top and on AK135 PKiKP amplitude at the bottom. Scale on top is PKiKP range (degrees) for a surface seismic event. Through most of its range, PKiKP amplitude is quite low, reaching unity where the $P$ wave that penetrates the core (PKIKP) reaches the critical incidence angle $\left(\theta \sim 70^{\circ}\right)$. Note PREM amplitudes change sign in the incidence angle $\theta$ range between $\sim 45^{\circ}$ and $55^{\circ}$, leading to logarithmic divergence. In contrast, AK135 amplitudes are always positive. Anisotropic reflectivity increases at near-vertical incidence and is enhanced by $10 \%$ levels of anisotropy, but at smaller levels the effect depends on the particular inner core boundary properties of PREM and AK135. Isotropic reflection coefficients calculated using the WKBJ synthetic algorithms (Chapman et al., 1988). PREM = Preliminary Reference Earth Model.

supplementary information for details on normalizing the count histogram to theoretical PKiKP amplitudes. The similarity between the PREM-predicted reflection amplitudes and the relative count levels at $25-45^{\circ}$ and $>120^{\circ}$ further supports the use of the reporting frequency count proxy for amplitude. There are systematic differences in reporting frequencies relative to PREM amplitudes evident in the range between $40^{\circ}$ and $120^{\circ}$, which will provide key diagnostics in the study.

\section{Results}

Figure 4 shows a typical amplitude versus incidence angle profile for PKiKP, using the properties of isotropic PREM and AK135 (Kennett et al., 1995). At an incidence angle of $\sim 70^{\circ}$, the amplitude jumps because a $P$ wave is no longer able to penetrate the solid. The $S$ wave speed in the inner core is much slower than the $P$ wave speed in the liquid outer core. Consequently, there are no cusps or amplitude anomalies due to $P$ or $S$ evanescence such as for ScP (Schweitzer, 2002). With hexagonal material at the surface of the ICB, however, additional effects arise. We parameterize the anisotropy in terms of the axial to equatorial change in $P$ wave speed, which yields the Thomsen parameter $\epsilon$ and let $\delta=2.9 \times \epsilon$ and $\gamma=3.4 \times \epsilon$. Figure 4 also shows the reflection intensity in the isotropic and anisotropic cases for the AK135 model and PREM. Of note is that at near-vertical incidence, the reflection coefficients are slightly larger, by up to a factor of 2 . Moreover, in the incidence range of $40-60^{\circ}$, reflection intensities vary substantially compared to the isotropic case. For PREM, anisotropy increases the reflectivity by factors of 2-10 as the $P$ anisotropy increases from $2.5 \%$ to $10 \%$. In contrast, for AK135, low $P$ anisotropy first decreases the reflectivity intensity, but then increases it, enhancing amplitudes by 10 at a $10 \%$ anisotropy level. At large incidence angles, $>70^{\circ}$, reflected wave amplitudes are insensitive to anisotropy.

Having established that anisotropy at the ICB substantially changes the PKiKP reflection coefficient, we now ask how much anisotropy is required to explain the amplitude trend with range as given by the PKiKP 


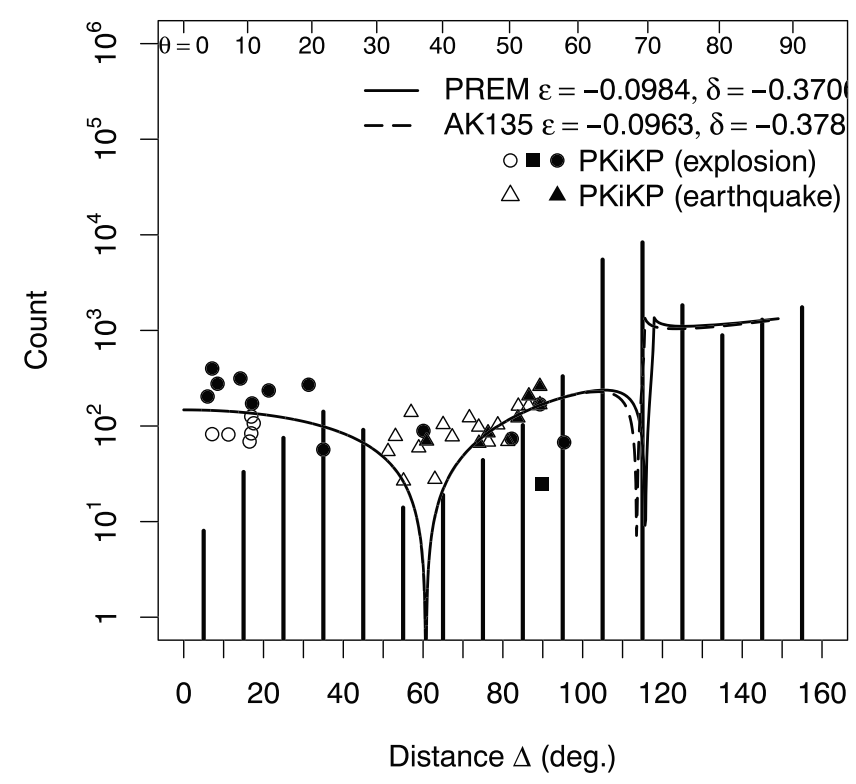

Figure 5. Best fitting anisotropy models for apparently isotropic PREM and AK135 ICB properties. Nuclear explosion amplitude data from Krasnoshchekov et al. (2005; filled: station averages; open: single observation), compared to isotropic predictions, and PKiKP/P amplitudes from Koper \& Dombrovskaya (2005; open: lower bound; filled: observed). Both amplitude studies show enhanced amplitudes in the distance range $\Delta$ of $60-100^{\circ}$ (Figure 4 ). See the supporting information for normalization details for the various data sources. PREM = Preliminary Reference Earth Model.

observation frequency proxy (Figure 3). To answer this question, we perform a grid search over all combinations of the Thomsen parameters $\epsilon, \delta$, and $\gamma$ in the range $-0.2 \leq \epsilon \leq 0,-0.4 \leq \delta \leq 0$, and $-0.4 \leq \gamma \leq 0$ over increments of 0.002 , followed by a nonlinear minimization of the best fit grid search combination to find the global minimum. We use the same normalization scheme described in section 2 to anchor the amplitudes to the PKiKP observation histogram, and use the difference between calculated and observed values between $55^{\circ}$ and $95^{\circ}$ and the value at $145^{\circ}$ as a misfit measure. No anisotropic model is able to reproduce the PKiKP histogram peak at $\sim 110^{\circ}$, which we suspect is due to difficulties in distinguishing PKiKP from $\mathrm{PP}$ and PKPdf here; hence, this distance range is excluded from the misfit measure. It emerges that $\gamma$ exerts a negligible influence on PKiKP (the observation frequencies change by $<0.1$ log unit and minima positions shift by $\pm 2^{\circ}$ ), so we report values for $\gamma=0$ even though systematics would suggest it is $\sim 3.9 \times \epsilon$.

Figure 5 shows the best fitting models using both PREM and AK135 ICB properties. Both models yield $P$ anisotropy levels around $10 \%$. The figure includes the PKIKP amplitude observations from nuclear explosions summarized in Krasnoshchekov et al. (2005), and the earthquake PKiKP amplitudes measured by Koper and Dombrovskaya (2005). The predicted anisotropic reflection amplitudes reproduce observations in the range between $80^{\circ}$ and $100^{\circ}$ better than purely isotropic models (Figure 4), but the data scatter is too large to draw any firm conclusions about the anisotropy level apart from the reporting frequency histogram.

\section{Discussion}

The main result, shown in Figure 4, is that anisotropy can substantially change the reflection coefficient of the $P$ wave reflected at the ICB and hence the amplitudes of PKiKP. The form of anisotropy we chose, VTI, yields PKPdf travel times in the shallow inner core that do not vary with spin axis orientation. Hence, the ICB surface that, to travel times, appears to be isotropic (Garcia \& Souriau, 2000; Ozounis \& Creager, 2001) might not in fact be so, an insight that motivated Lythgoe \& Deuss's (2015) study. Rather, solid crystallites might be oriented by compaction, flow, or electromagnetic forces uniformly upon adhesion to the ICB that could impart a uniform VTI fabric.

Why might the strength of the fabric vary longitudinally, as implied by the results of Creager (1999), Garcia and Souriau (2000), and Creager (2000)? Compaction rate might be a factor, because this is the alignment process in our VSPC simulations. The initial degree of alignment at the ICB surface could also vary 
due to flow variations in the outer core that affect either the adhesion of crystallites to the surface or the light-element concentration that would affect surface growth. Explaining the variable thickness of the anisotropic region is harder. The process that realigns the anisotropy with the inner core's bulk is likely to be an internal one such as convective flow or an electromagnetic force whose strength varies radially. One could speculatively appeal to a hemispherically asymmetric convective flow that entails thinning of the boundary layer at the ICB or a hemispheric change in magnetic field structure that mediates crystal realignment. Whatever its cause, an aligned anisotropic layer around the ICB seems incompatible with lateral translation of the inner core (Alboussière et al., 2010; Monnereau et al., 2010) which, if it was occurring, would require that highly aligned crystals result both from the approach to melting conditions in the melting hemisphere and to freezing conditions in the crystallizing hemisphere.

Cormier (2007) found that the fabric at the surface of the ICB appears to be anisotropic, being elongated radially in the equatorial Eastern Hemisphere, whereas it appears elongated circumferentially in the Western Hemisphere. This might create seismic anisotropy by shape preferred orientation (as distinct from the crystal preferred orientation model we use), and a hemispheric modulation of the anisotropy's strength could arise. The inhomogeneous PKiKP reflection point distribution (supporting information Figure S5), particularly in the range $50 \leq \Delta \leq 90$ where anisotropy's effect is strongest on amplitudes, makes any fabric effect hard to assess. There is some suggestion that the far eastern hemisphere is more strongly anisotropic, but it could also be due to geographic source-receiver bias (Figure S1).

While the range dependence of the isotropic theoretical PKiKP reflection coefficients differ substantially between PREM and AK135 (Figure 4), it is remarkable how the changes due to anisotropy can modify them to match the proxy range dependence (Figure 5). This shows that anisotropy has a first-order effect on amplitudes, as important as density and wave speed.

The same form of anisotropy also substantially solves the conundrum of unexpectedly high observed PKiKP amplitudes (Koper \& Dombrovskaya, 2005; Krasnoshchekov et al., 2005). Anisotropy boosts the amplitude of the reflected $P$ wave at the ICB by factors up to 10 times the expected one at incidence angles of 40-50, where isotropic models predict low amplitudes (Figure 4) and where PKiKP is frequently reported (Figure 5). A glassy outer core near the ICB (Cormier, 2009) explains the high amplitudes equally well but requires new physics in the liquid state to emerge due to changes in liquid composition. Our model has the virtue of not requiring any new properties of the inner or outer core, simply the evolution of a known solid property, the inner core's anisotropy, as the core crystallizes. Similarly, lateral changes in the ICB's properties (Attanayake et al., 2018; Krasnoshchekov et al., 2005) or topography (Cao et al., 2007; de Silva et al., 2017) may also modulate PKiKP amplitudes, but anisotropy's first-order effect on amplitudes suggests that a parsimonous approach would first attribute amplitude changes to changes in anisotropy to before invoking other effects.

Anisotropy is, however, no panacea. It is clear that even ICB anisotropy fails to explain reflection amplitudes close to $60^{\circ} \Delta$. The discrepancy may be evidence for a need to revise the isotropic properties of the ICB. Koper and Dombrovskaya (2005) showed that zeros in the PKiKP amplitude versus distance curve are strongly affected by small changes in $P$ wave speed (5-10\%), density (3-5\%), and by more substantial changes in $S$ speed $(\sim 50 \%)$. Around $60^{\circ}$, the discrepancy between observed and predicted amplitudes with anisotropy might provide further constraints on the ICB's isotropic properties.

One valid criticism of the model is the strength of the anisotropy: It is around 10\% rather than the $~ 3 \%$ anisotropy found in the bulk of the inner core determined in other travel time studies. Single-crystal anisotropy is generally much larger than polycrystalline anisotropy due to imperfect crystal alignment (Barruol \& Mainprice, 1993), and it is impossible that the inner core is a giant single crystal due to the radial and hemispheric variation in isotropic and anisotropic properties (Song \& Helmberger, 1998; Tanaka \& Hamaguchi, 1997). Hence, the $\sim 3 \%$ bulk anisotropy should be lower than any single-crystal value. If the alignment mechanism operating at the ICB that creates VTI is more effective than the solid state fabric evolution that forms the bulk anisotropy, the surface anisotropy could more closely approximate the single-crystal values. We also note that these are the best fit values, but anisotropy levels as low as 5\% also rank among the lowest $0.1 \%$ of the misfit values in the grid search range explored. An adequate model could have significantly lower anisotropy than $10 \%$, in the $2-5 \%$ range compatible with inner core normal mode frequency shifts (Lythgoe \& Deuss, 2015). 
We use the frequency of observations of PKiKP as a proxy for PKiKP amplitude variation with distance, and, equivalently, incidence angle at the ICB. Most of the midrange data, which constrain the strength of the anisotropy, come from the Eastern Hemisphere. Use of the proxy is an expedient used in lieu of good amplitude measurements such as Krasnoshchekov et al.'s (2005) and Koper \& Dombrovskaya's (2005) over the whole range that PKiKP exists. Amplitude measurements are rare and even then prone to scatter (Figure 5). Skeptics will feel that the observation frequencies are doubly imperfect, being both an approximate estimator and one for an intrinsically noisy wavefield attribute. The shape of the range dependence is the key feature, however, and bears some resemblance to the sparse amplitude measurements, lending it some credibility. Hence, despite its flaws, the amplitude proxy is a useful fitting benchmark but should not be viewed as yielding a definitive estimate of anisotropy. Rather, it demonstrates the viability of a VTI model for the outermost inner core.

Our grid search could be extended to include the ICB density contrast, which is difficult to constrain seismically (Masters \& Gubbins, 2003). It would be tempting to use the amplitude constraints we have to improve existing inner core density models. We refrained from doing this, however, in order to show in a simple way how strong the effect of anisotropy is on ICB reflection amplitudes. There are likely to be strong tradeoffs between density and anisotropy due to their both having a first-order effect on amplitudes. Until a reliable set of amplitude observations is available, we believe the tradeoffs involved would not lead to a better understanding of the properties of the inner core.

\section{Conclusions}

We showed that the inner core's apparently isotropic outermost layer might actually be anisotropic, but with hexagonal symmetry with a radially directed symmetry axis. Models of weak $(<20 \%)$ anisotropy can lead to order-of-magnitude increases in $P$ wave reflections off of the ICB, observed at the Earth's surface as PKiKP at levels as low as $2-10 \%$ depending on the model. Using a proxy for the range dependence of PKiKP amplitudes, we found an anisotropy model that closely approximates the range dependence, which is quite different to the predicted reflection coefficients for an isotropic ICB. The best fit model has 5-10\% $P$ wave anisotropy, which could evolve into the bulk $3 \%$ anisotropy of the inner core as its texture develops over time.

\section{Acknowledgments}

We acknowledge, with thanks, Dmitry Krasnoschekov, whose study of the mosaic structure of the inner core prompted this one. The reviewers' insights led to some key clarifications to the manuscript, for which we thank them. This work partially funded by JSPS Kakenhi "Core-Mantle Co-Evolution" project, and MEXT Kakenhi Grant 15 H05832 to G. H. Except for the VPSC modeling and isotropic reflection coefficient calculations, all other calculations and all figures were made using $\mathrm{R}$ ( $\mathrm{R}$ Core Team, 2017). ISC data available online from http://www.isc.ac.uk (ISC Bulletin: arrivals search).

\section{References}

Alboussière, T., Deguen, R., \& Melzani, M. (2010). Melting-induced stratification above the Earth's inner core due to convective translation. Nature, 466, 744-747.

Attanayake, J., Thomas, C., Cormier, V. F., Miller, M. S., \& Koper, K. D. (2018). Irregular transition layer beneath the Earths inner core boundary from observations of antipodal PKIKP and PKIIKP Waves. Geochemistry, Geophysics, Geosystems, 19, 3607-3622. https://doi. org/10.1029/2018GC007562

Barruol, G., \& Mainprice, D. (1993). 3-D seismic velocities calculated from lattice-preferred orientation and reflectivity of a lower crustal section: Examples of the Val Sesia section (Ivrea zone, northern Italy). Geophysical Journal International, 115, 1169-1188.

Cao, A., Masson, Y., \& Romanowicz, B. (2007). Short wavelength topography on the inner-core boundary. Proceedings of the National Academy of Sciences of the United States of America, 104, 31-35.

Chapman, C. H., Jen-yi, C., \& Lyness, D. G. (1988). The WKBJ seismogram algorithm. In D. J. Doornbos (Ed.), Seismological algorithms (pp. 469+xviipp). San Diego: Academic Press.

Chapuis, A., \& Driver, J. H. (2011). Temperature dependency of slip and twinning in plane strain compressed magnesium single crystals. Acta Materialia, 59, 1986-1994.

Cormier, V. F. (2007). Texture of the uppermost inner core from forward- and back-scattered seismic waves. Earth and Planetary Science Letters, 258, 442-453.

Cormier, V. F. (2009). A glassy lowermost outer core. Geophysical Journal International, 179, 374-380.

Creager, K. C. (1992). Anisotropy of the inner core from differential travel-times of the phases PKP and PKIKP. Nature, 356, 309-314.

Creager, K. C. (1999). Large-scale variations in inner core anisotropy. Journal of Geophysical Research, 104, 23,127-23,139.

Creager, K. C. (2000). Inner core anisotropy and rotation. In S.-I. Karato, A. Forte, R. Lieberman, G. Masters, \& L. Stixrude (Eds.), Earth's deep interior: Mineral physics and tomography from the atomic to the global scale (Vol. 117, pp. 89-114). Washington DC: American Geophysical Union.

de Silva, S., Cormier, V. F., \& Zheng, Y. (2017). Inner core boundary topography explored with reflected and diffracted P waves. Physics of the Earth and Planetary Interiors, 276, 202-214.

Deguen, R. (2012). Structure and dynamics of Earth's inner core. Earth and Planetary Science Letters, 333-334, $211-225$.

Dziewonski, A., \& Anderson, D. (1981). Preliminary reference Earth model. Physics of the Earth and Planetary Interiors, 25, 297-356.

Garcia, R., \& Souriau, A. (2000). Inner-core anisotropy and heterogeneity level. Geophysical Research Letters, 27, 3121-3124.

Giardini, D., Woodhouse, J. H., \& Li, X. D. (1987). Three-dimensional structure of the Earth from splitting in free-oscillation spectra. Nature, 325, 405-411.

Kennett, B. L. N., Engdahl, E. R., \& Buland, R. (1995). Constraints on seismic velocities in the Earth from traveltimes. Geophysical Journal International, 122, 108-124.

Koper, K. D., \& Dombrovskaya, M. (2005). Seismic properties of the inner core boundary from PKiKP/P amplitude ratios. Earth and Planetary Science Letters, 237, 680-694. 
Krasnoshchekov, D. N., Kaazik, P. B., \& Ovtchinnikov, V. M. (2005). Seismological evidence for mosaic structure of the surface of the Earth's inner core. Nature, 435, 483-487.

Lebensohn, R. A., \& Tomé, C. N. (1993). A self-consistent anisotropic approach for the simulation of plastic deformation and texture development of polycrystals: Application to zirconion alloys. Acta Metallurgica et Materialia, 41, 2611-2624.

Li, Y., Vočadlo, L., \& Brodholt, J. P. (2018). The elastic properties of hcp-Fe alloys under the conditions of the Earth's inner core. Earth and Planetary Science Letters, 493, 118-127.

Lythgoe, K. H., \& Deuss, A. (2015). The existence of radial anisotropy in Earth's upper inner core revealed from seismic normal mode observations. Geophysical Research Letters, 42, 4841-4848. https://doi.org/10.1002/2015GL064326

Mainprice, D., Bachmann, F., Hielscher, R., \& Schaeben, H. (2015). Descriptive tools for the analysis of texture projects with large datasets using MTEX: Strength, symmetry and components. In D. R. Faulkner, J. Meckenburgh, \& E. Mariani (Eds.), Rock deformation from field, experiments and theory: A volume in honour of Ernie Rutter (Vol. 409, pp. 251-271). London: Geological Society.

Malehmir, R., \& Schmitt, D. R. (2016). ARTc: Anisotropic reflectivity and transmissivity calculator. Computers \& Geosciences, 93, $114-126$.

Masters, G., \& Gubbins, D. (2003). On the resolution of density within the Earth. Physics of the Earth and Planetary Interiors, 140, 159-167.

Monnereau, M., Calvet, M., Margerin, L., \& Souriau, A. (2010). Lopsided growth of Earth's inner core. Science, 328, 1014-1017.

Morelli, A., Dziewonski, A., \& Woodhouse, J. H. (1986). Anisotropy of the inner core inferred from PKIKP travel-times. Geophysical Research Letters, 13, 1545-1548.

Ozounis, A., \& Creager, K. C. (2001). Isotropy overlying anisotropy at the top of the inner core. Geophysical Research Letters, 28, 4331-4334. Poupinet, G., Pillet, R., \& Souriau, A. (1983). Possible heterogeneity of the Earth's core deduced from PKIKP travel-times. Nature, 305, 204-206.

R Core Team (2017). R: A language and environment for statistical computing. Vienna, Austria: R Foundation for Statistical Computing. https://www.R-project.org/

Ritzwoller, M., Masters, G., \& Gilbert, F. (1986). Observations of anomalous splitting and their interpretation in terms of aspherical structure. Journal of Geophysical Research, 91, 203-228.

Schweitzer, J. (2002). Simultaneous inversion of steep-angle observations of PcP and ScP in Europe - What can we learn about the core-mantle boundary? Geophysical Journal International, 151, 209-220.

Shearer, P. M. (1994). Constraints on inner-core anisotropy from PKP(DF) travel-times. Journal of Geophysical Research, 99, 19,647-19,659. Song, X. D., \& Helmberger, D. V. (1993). Anisotropy of Earth's inner-core. Geophysical Research Letters, 20, 2591-2594.

Song, X. D., \& Helmberger, D. V. (1998). Seismic evidence for an inner core transition zone. Science, 282, 924-927.

Steinle-Neumann, G., Stixrude, L., \& Cohen, R. E. (2001). Elasticity of iron at the temperature of the Earth's inner core. Nature, 413, 57-60. Stixrude, L., \& Cohen, R. E. (1995). High-pressure elasticity of iron and anisotropy of Earth's inner core. Science, 267, 1972-1975.

Sumita, I., \& Olson, P. (2002). Rotating thermal convection experiments in a hemispherical shell with heterogeneous boundary heat flux: Implications for the Earth's core. Journal of Geophysical Research, 107(B8), 2169. https://doi.org/10.1029/2001JB000548

Tanaka, S., \& Hamaguchi, H. (1997). Degree one heterogeneity and hemispherical variation of anisotropy in the inner core from PKP(BC)-PKP(DF) times. Journal of Geophysical Research, 102, 2925-2938.

Thomsen, L. (1986). Weak elastic anisotropy. Geophysics, 51, 1954-1966.

Tkalčić, H. (2015). Complex inner core of the Earth: The last frontier of global seismology. Reviews of Geophysics, 53, 59-94. https://doi. org/10.1002/2014RG000469

Woodhouse, J. H., Giardini, D., \& Li, X. D. (1986). Evidence for inner core anisotropy from free oscillations. Geophysical Research Letters, 13, 1549-1552. 